\title{
Are There Sex-Specific Differences in Response to Adjunctive Host- Directed Therapies for Tuberculosis?
}

\begin{abstract}
Noton K. Dutta ${ }^{1 *}$ and Bianca E. Schneider ${ }^{2}$
${ }^{1}$ Center for Tuberculosis Research, Department of Medicine, Johns Hopkins University School of Medicine, Baltimore, MD, United States, ${ }^{2}$ Junior Research Group Coinfection, Priority Research Area Infections, Research Center Borstel - Leibniz Lung Center, Borstel, Germany
\end{abstract}

Keywords: Mycobacterium tuberculosis, tuberculosis, host directed therapy, statins, treatment outcome, sexspecific differences, male-bias, mouse models

\section{INTRODUCTION}

Tuberculosis (TB) appears to afflict men more than women, but the underlying reasons for this disparity and whether there are sex-based differences in TB treatment responses are unknown. Based on a knowledge gap in previously published studies, a pertinent research question is being asked: Are there sex-specific differences in response to adjunctive host-directed therapy (HDT) for TB? Using statins as a prototype, we also highlight the incorporation of appropriately designed studies on sex differences in HDT.

There is an important need for new drugs to help control the TB pandemic, counteract the emergence of drug resistance worldwide, and supplement the limited number of antimicrobials that are currently in the TB drug discovery pipeline. Therefore, the repurposing of existing drugs, including host-directed therapies (1), is being investigated as a means of accelerating the development of novel TB regimens in the clinical setting. We hypothesize that there is a sex difference in responses to adjunctive HDT because males are more susceptible to TB and various HDTs may have differential, sex-dependent impacts on inflammation. This question is important in the context of ongoing clinical trials that are investigating the potential roles of various HDTs as adjunctive therapy for TB, including the lipid-lowering agents, statins (StAT-TB, NCT03882177), the hypoglycemic agent, metformin (CTRI/2018/01/011176), and the anticancer agent, imatinib (IMPACT-TB, NCT03891901). Mayer-Barber and colleagues reported that the 5-lipoxygenase inhibitor zileuton, which is FDA-approved for the treatment of asthma, increased prostaglandin E2 (PGE2) levels in the lungs of Mycobacterium tuberculosis-infected mice and prevent acute mortality, while decreasing pulmonary bacillary loads, tissue necrosis, and type I IFN levels (2). Data from Pace et al. suggested that zileuton is less effective in males, prompting consideration of sex-based differences in leukotriene biosynthesis blockade for respiratory and cardiovascular diseases (3).

\section{INCREASED TB INCIDENCE IN MEN}

Despite over a century of research, TB still kills $\sim 1.5$ million people every year (4). Globally, TB notification data show a male-to-female ratio of bacteriologically-confirmed pulmonary TB ranging from 1.2 in Ethiopia to 4.9 in Viet Nam (4), but the underlying reasons for this male bias remain elusive. Presumably, both gender- and sex-related factors contribute to higher TB rates in men. Socioeconomic and cultural factors influence exposure and help-seeking behavior, and confounding factors, such as smoking, alcohol and drug use, which are known risk factors for $\mathrm{TB}$, are more common in men $(5,6)$. However, these factors are unlikely to explain the consistent global male bias, and biological 
differences between the sexes likely affect susceptibility to mycobacterial infection and disease outcome $(7,8)$. Despite the well-known sex-based differences in human TB incidence rates, due to practical considerations, most animal studies have either used only one sex or do not report the sex of the animals at all.

\section{THE ROLE OF BIOLOGICAL SEX IN TB}

Recent data suggest that male C57BL/6 mice are less able to control $M$. tuberculosis infection relative to their female counterparts due to impaired innate and adaptive immune responses, as manifested by increased lung bacillary burdens and accelerated death (9). Divergent innate immune priming events occurring early in an infection influence both the bacterial load "set-point" and the ensuing adaptive immune response (10).

Innate recognition of pathogens and the induction of inflammatory and antimicrobial immune responses differ between the sexes (11). Factors that have been shown to account for the sex-based disparity in immune responses include genetic factors and hormonal mediators. Estrogen was shown to boost the ability of macrophages to kill Streptococcus pneumoniae (12), and the antimicrobial activity of peritoneal macrophages from female mice was significantly more potent against Mycobacterium intracellulare than that of male mice (13). IFN $\gamma$, a critical cytokine mainly produced by $\mathrm{CD}^{+}$and $\mathrm{CD}^{+} \mathrm{T}$ cells, increases the anti-bacterial effector functions of macrophages (14). Invariant natural killer $\mathrm{T}$ (iNKT) cells are a subset of $\mathrm{T}$ cells that bridge innate and adaptive immunity, recognize $M$. tuberculosis-infected macrophages, produce IFN $\gamma$, and kill intracellular bacteria (15). Estradiol induces iNKT cells from female mice to produce more IFN $\gamma$ than their male counterparts (16). Moreover, IFN $\gamma$ production by iNKT cells is impaired by female castration or genetic ablation of the estradiol receptor, and the normal phenotype is restored by estradiol injection $(16,17)$ In contrast, testosterone was shown to exert anti-inflammatory effects in macrophages and other innate immune cells that express the androgen receptor (AR), by inhibiting pro-inflammatory factors such as TNF- $\alpha$ and nitric oxide (NO), and increasing the synthesis of interleukin (IL)-10 (18). Male castration also increases TNF- $\alpha$ secretion in macrophages (19). These results illustrate the general, but probably simplistic, perception of estradiol as an immunitysustaining or immunity-enhancing mediator, and of testosterone as a mediator that inhibits the immune response. Similarly, male mice are more susceptible to TB than female mice, which can be prevented by male castration (20), suggesting that testosterone could be a TB susceptibility factor. Sex hormones not only regulate innate but also adaptive responses. Generally, females show higher $\mathrm{T}$ cell activity including the production of $\mathrm{T}_{\mathrm{h}} 1$-type cytokines such as IFN $\gamma$, and greater antibody responses than males (11). Differences in adaptive immunity might contribute to the impaired long-term containment of $M$. tuberculosis observed in mice $(9,20,21)$. Indeed, we recently showed that increased male susceptibility in $M$. tuberculosis infection is associated with reduced $\mathrm{B}$ cell follicle formation in the lung (21).
Sex differences in $\mathrm{TB}$ can be mediated by more than hormonal influence. The $\mathrm{X}$ chromosome expresses a number of immune-related genes, as well as a number of immuneassociated microRNAs (11). Females have two X chromosomes and benefit from a genetic diversity due to cellular mosaicism and genes escaping $\mathrm{X}$ chromosome inactivation which is often advantageous because it ameliorates the deleterious effects of Xlinked mutations. Thus, sex disparity in TB may be a result of both hormonal and genetic influences.

\section{STATINS AS ADJUNCTIVE HDT FOR TB}

In addition to their cholesterol-lowering properties, $\beta$-Hydroxy $\beta$-methylglutaryl-CoA (HMG-CoA) reductase inhibitors (statins) have been shown to have broad anti-inflammatory and immunomodulatory properties $(22,23)$, and their use has been associated with significantly decreased risk of TB (24). Statins have been shown to have antimicrobial and immunomodulatory activity in mouse models of infection against intracellular pathogens, including Salmonella enterica and Chlamydia pneumoniae. Statins' mode of action in antimicrobial therapy is centered on controlling the infection rate in macrophages and monocytes. Mature macrophages respond to diverse environmental signals by expressing many functional phenotypes, from the classical phenotype (M1, proinflammatory) to the alternative phenotype (M2, antiparasitic, immunoregulatory). Early secreted antigenic target of $6-\mathrm{kDa}$ of $M$. tuberculosis induces M1 phenotype in the early stage and then polarizes to M2 phenotype in the later stage of TB infection (25). Heat-Shock Protein 16.3 of M. tuberculosis induces M2 polarization in the mouse bone marrow-derived macrophage model via CCRL2/CX3CR1 and may be mediated by the AKT/ ERK/p38-MAPK signaling pathway. In fact, adoptive transfer of M2 macrophages is effective in controlling TB infection apart from its role in controlling tissue damage (26)

In a recent publication (27), we screened eight different statins for a cytotoxic effect, anti-tubercular activity, synergy with firstline drugs in macrophages, pharmacokinetics, and adjunctive bactericidal activity in two different mouse models as a potential adjunctive therapy to existing first-line TB drugs (27). Pravastatin exhibited the most favorable therapeutic index in vitro and better anti-TB activity in the standard BALB/c mouse model and in the $\mathrm{C} 3 \mathrm{HeB} / \mathrm{FeJ}$ mouse model of human-like necrotic TB lung granulomas (27). Previous studies already demonstrated that pravastatin modulated phagosomal maturation characteristics in macrophages via phenocopying macrophage activation, and its use as an adjunctive agent in chronically infected mice altered lung and peripheral immune responses $(27,28)$. We also found that simvastatin adjunctive therapy enhanced the first-line TB regimen's antimicrobial activity and shortened the time required to achieve cure in a BALB/c mouse model of chronic TB infection (29). Bruiners et al. recently demonstrated that simvastatin inhibits mechanistic target of rapamycin complex 1 (mTORC1) activity and regulates transcription factor EB (TFEB) nuclear translocation to induce autophagy and lysosomal biogenesis (30). In addition, statins show synergy with the key sterilizing drug, 


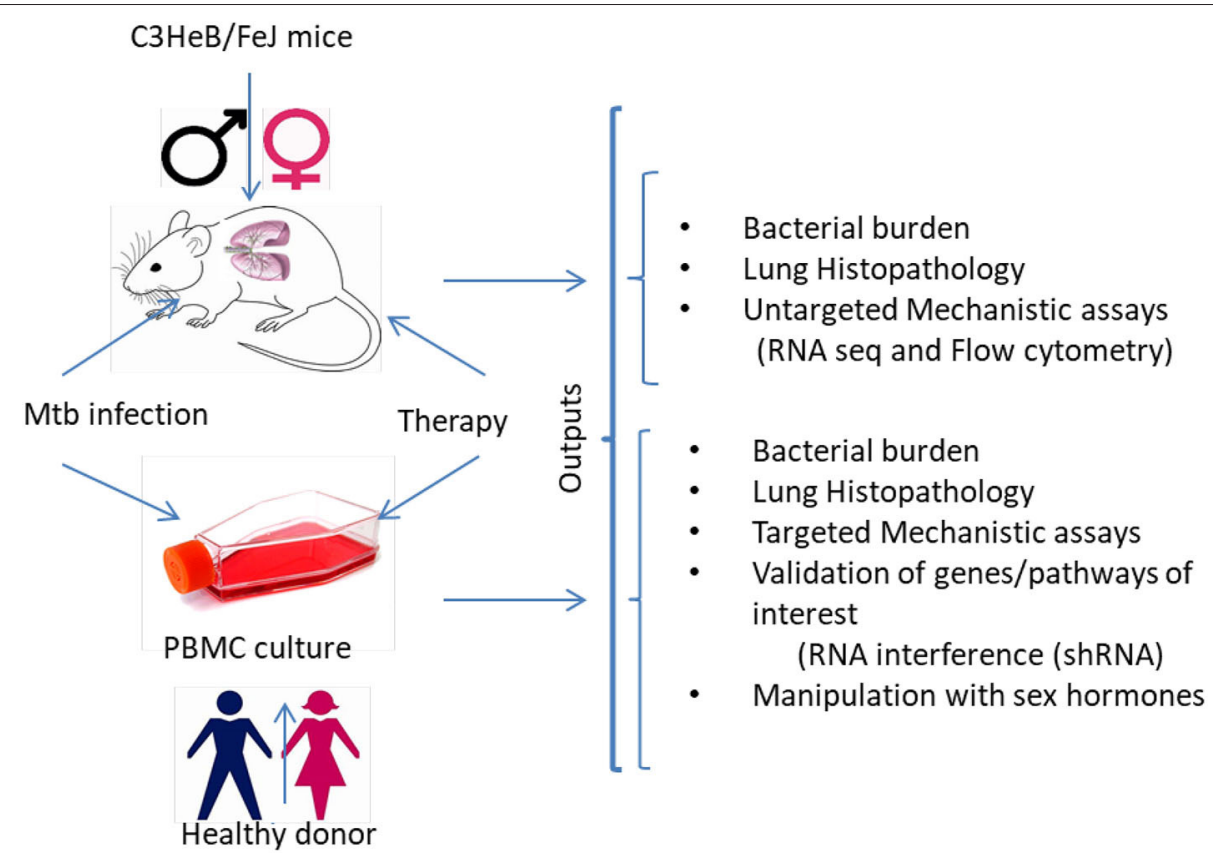

FIGURE 1 | Experimental sketch for preclinical examination of sex-differences in responsiveness to HDTs. The experimental strategy would help to determine sex-based differences in clinical, microbiological, immunological and histopathological outcomes in M. tuberculosis infected (i) C3HeB/FeJ male and female mice and (ii) human monocyte-derived macrophages from healthy male and female donors before and after treatment with HDTs. Several untargeted and targeted approaches can be used to investigate the molecular mechanisms associated with the sex-specific anti-TB activity of HDTs. MDMs, monocyte-derived macrophages; shRNA, Small hairpin RNA.

rifampin. Retrospective cohort studies found that statin use was associated with a reduced incidence of active TB disease $(31,32)$. However, studies focusing on anti-TB effect of statin on the basis of sex of the patients are lacking. Also, the preclinical studies were conducted exclusively in female mice, and it is unknown whether the adjunctive, host-directed anti-TB properties of statins are sexspecific, which is an important consideration for their potential clinical utility.

\section{SEX DIFFERENCES IN THE EFFECTIVENESS OF STATINS}

Previous studies (33) have shown sex-based differences for statins with respect to mortality following myocardial infarction (34) and in reducing the risk of Alzheimer's disease (35). These effects may result from differences in drug metabolism, but hormonal effects have not been explored. In some animal studies, the simvastatin metabolism rate was found to be considerably higher in males than in females (36); this statin might, therefore, be expected to have a greater clinical effect in males. This hypothesis was not confirmed in studies that enrolled human volunteers, while, in contrast, a lower rate of simvastatin and lovastatin metabolism was observed in men than in women (37). Moreover, several epidemiological studies have reported greater statininduced reductions in both LDL and total cholesterol in women than in men (38).

\section{CONCLUSIONS AND FUTURE DIRECTIONS}

It has already been established that sex must be considered in preclinical studies, although clearly defined "go/no-go" endpoints to justify further testing of various HDT agents in clinical trials have yet to be defined. It is unknown if the antiTB activity of statins and other promising HDTs are sex specific. The study of animals and cells of both sexes is essential to include preclinical study designs that will control drug exposure, efficacy, metabolism, and immune response variabilities on HDT for TB. Here, we propose to understand if sex influences the adjunctive anti-TB activity and immune responses of HDTs in: (i) a murine model of chronic TB infection with humanlike necrotic lung granulomas; and (ii) ex vivo infection of human monocyte-derived macrophages (MDMs) (Figure 1). M. tuberculosis residing in necrotic mouse lung lesions may be more akin to persisters in human lesions with a reduced response to direct-acting anti-TB drugs; further, these areas represent relative pharmacological sanctuaries. Because of these favorable features, we and other groups have begun to use $\mathrm{C} 3 \mathrm{HeB} / \mathrm{FeJ}$ mice to test the efficacy of various antitubercular regimens and novel anti-inflammatory therapies $(27,39)$. An MDM system was chosen because macrophages are the key cell type harboring $M$. tuberculosis during infection and because this model is amenable to testing under multiple, controlled perturbations. Moreover, circulating monocytes are natural precursors of lung tissue-resident macrophages. In addition, 
using primary cells, researchers will be able to validate the key findings in vivo in macrophages from both males and females by applying RNA interference technology. Downstream "omics" data will provide the opportunity to investigate the mechanisms underlying sex-based differences in host control of $M$. tuberculosis infection, as well as potential differences in response to standard antitubercular therapy and HDT between the sexes. Such results will improve the predictive value of animal models to evaluate treatment efficacy by HDT agents with respect to variables such as sex and potential clinical utility of particular immunomodulators. The identification of the biological pathways underlying sex differences in HDTs for TB

\section{REFERENCES}

1. Frank DJ, Horne DJ, Dutta NK, Shaku MT, Madensein R, Hawn TR, et al. Remembering the host in tuberculosis drug development. J Infect Dis. (2019) 219:1518-24. doi: 10.1093/infdis/jiy712

2. Mayer-Barber KD, Andrade BB, Oland SD, Amaral EP, Barber DL, Gonzales J, et al. Host-directed therapy of tuberculosis based on interleukin-1 and type I interferon crosstalk. Nature. (2014) 511:99-103. doi: 10.1038/nature13489

3. Pace S, Pergola C, Dehm F, Rossi A, Gerstmeier J, Troisi F, et al. Androgenmediated sex bias impairs efficiency of leukotriene biosynthesis inhibitors in males. J Clin Invest. (2017) 127:3167-76. doi: 10.1172/JCI92885

4. WHO. Global Tuberculosis Report 2019. Geneva: World Health Organization. (2019).

5. Neyrolles O, Quintana-Murci L. Sexual inequality in tuberculosis. PLoS Med. (2009) 6:e1000199. doi: 10.1371/journal.pmed.1000199

6. Hargreaves JR, Boccia D, Evans CA, Adato M, Petticrew M, Porter JD. The social determinants of tuberculosis: from evidence to action. Am J Public Health. (2011) 101:654-62. doi: 10.2105/AJPH.2010.199505

7. Nhamoyebonde S, Leslie A. Biological differences between the sexes and susceptibility to tuberculosis. J Infect Dis. (2014) 209 (Suppl. 3):S100-106. doi: 10.1093/infdis/jiu147

8. Hertz D, Schneider B. Sex differences in tuberculosis. Semin Immunopathol. (2019) 41:225-37. doi: 10.1007/s00281-018-0725-6

9. Dibbern J, Eggers L, Schneider BE. Sex differences in the C57BL/6 model of Mycobacterium tuberculosis infection. Sci Rep. (2017) 7:10957. doi: 10.1038/s41598-017-11438-z

10. Chamekh M, Deny M, Romano M, Lefevre N, Corazza F, Duchateau J, et al. Differential susceptibility to infectious respiratory diseases between males and females linked to sex-specific innate immune inflammatory response. Front Immunol. (2017) 8:1806. doi: 10.3389/fimmu.2017.01806

11. Klein SL, Flanagan KL. Sex differences in immune responses. Nat Rev Immunol. (2016) 16:626-38. doi: 10.1038/nri.2016.90

12. Yang Z, Huang YC, Koziel H, De Crom R, Ruetten H, Wohlfart P, et al. Female resistance to pneumonia identifies lung macrophage nitric oxide synthase- 3 as a therapeutic target. Elife. (2014) 3:e03711. doi: 10.7554/eLife.03711.013

13. Yamamoto $Y$, Tomioka $H$, Sato K, Saito H, Yamada Y, Setogawa T. Sex differences in the susceptibility of mice to infection induced by Mycobacterium intracellulare. Am Rev Respir Dis. (1990) 142:430-3. doi: 10.1164/ajrccm/142.2.430

14. Kak G, Raza M, Tiwari BK. Interferon-gamma (IFN-gamma): exploring its implications in infectious diseases. Biomol Concepts. (2018) 9:64-79. doi: 10.1515/bmc-2018-0007

15. Sada-Ovalle I, Chiba A, Gonzales A, Brenner MB, Behar SM. Innate invariant NKT cells recognize Mycobacterium tuberculosis-infected macrophages, produce interferon-gamma, and kill intracellular bacteria. PLoS Pathog. (2008) 4:e1000239. doi: 10.1371/journal.ppat.1000239

16. Gourdy P, Araujo LM, Zhu R, Garmy-Susini B, Diem S, Laurell H, et al. Relevance of sexual dimorphism to regulatory $\mathrm{T}$ cells: estradiol promotes IFN-gamma production by invariant natural killer T cells. Blood. (2005) 105:2415-20. doi: 10.1182/blood-2004-07-2819 will play an essential role in the development of more effective personalized healthcare. One unanswered question is to what extent the mouse model will be predictive of HDTs for TB in humans.

\section{AUTHOR CONTRIBUTIONS}

ND and BS have made substantial, direct and intellectual contribution to the work, critically reading an earlier version of this manuscript, and approved it for publication. All authors contributed to the article and approved the submitted version.

17. Janele D, Lang T, Capellino S, Cutolo M, Da Silva JA, Straub RH. Effects of testosterone, 17beta-estradiol, and downstream estrogens on cytokine secretion from human leukocytes in the presence and absence of cortisol. Ann N Y Acad Sci. (2006) 1069:168-82. doi: 10.1196/annals.1351.015

18. D’agostino P, Milano S, Barbera C, Di Bella G, La Rosa M, Ferlazzo V, et al. Sex hormones modulate inflammatory mediators produced by macrophages. Ann N Y Acad Sci. (1999) 876:426-9. doi: 10.1111/j.1749-6632.1999.tb07667.x

19. Rettew JA, Huet-Hudson YM, Marriott I. Testosterone reduces macrophage expression in the mouse of toll-like receptor 4, a trigger for inflammation and innate immunity. Biol Reprod. (2008) 78:432-7. doi: 10.1095/biolreprod.107.063545

20. Bini EI, Mata Espinosa D, Marquina Castillo B, Barrios Payan J, Colucci D, Cruz AF, et al. The influence of sex steroid hormones in the immunopathology of experimental pulmonary tuberculosis. PLoS ONE. (2014) 9:e93831. doi: 10.1371/journal.pone.0093831

21. Hertz D, Dibbern J, Eggers L, Von Borstel L, Schneider BE. Increased male susceptibility to Mycobacterium tuberculosis infection is associated with smaller B cell follicles in the lungs. Sci Rep. (2020) 10:5142. doi: 10.1038/s41598-020-61503-3

22. Hennessy E, Adams C, Reen FJ, O'gara F. Is there potential for repurposing statins as novel antimicrobials? Antimicrob Agents Chemother. (2016) 60:5111-21. doi: 10.1128/AAC.00192-16

23. Dutta NK, Karakousis PC. Reply to Hu et al: Could there be detrimental effects of statin adjunctive TB therapy on immune responses? J Infect Dis. (2019). doi: 10.1093/infdis/jiz676. [Epub ahead of print].

24. Duan H, Liu T, Zhang X, Yu A, Cao Y. Statin use and risk of tuberculosis: a systemic review of observational studies. Int J Infect Dis. (2020) 93:168-74. doi: 10.1016/j.ijid.2020.01.036

25. Refai A, Gritli S, Barbouche MR, Essafi M. Mycobacterium tuberculosis virulent factor ESAT-6 drives macrophage differentiation toward the proinflammatory M1 phenotype and subsequently switches it to the antiinflammatory M2 phenotype. Front Cell Infect Microbiol. (2018) 8:327. doi: $10.3389 /$ fcimb. 2018.00327

26. Pineros AR, Campos LW, Fonseca DM, Bertolini TB, Gembre AF, Prado RQ, et al. M2 macrophages or IL-33 treatment attenuate ongoing Mycobacterium tuberculosis infection. Sci Rep. (2017) 7:41240. doi: 10.1038/srep41240

27. Dutta NK, Bruiners N, Zimmerman MD, Tan S, Dartois V, Gennaro ML, et al. Adjunctive host-directed therapy with statins improves tuberculosis-related outcomes in mice. J Infect Dis. (2019) 221:1079-87. doi: 10.1093/infdis/jiz517

28. Parihar SP, Guler R, Khutlang R, Lang DM, Hurdayal R, Mhlanga MM, et al. Statin therapy reduces the Mycobacterium tuberculosis burden in human macrophages and in mice by enhancing autophagy and phagosome maturation. J Infect Dis. (2014) 209:754-63. doi: 10.1093/infdis/jit550

29. Dutta NK, Bruiners N, Pinn ML, Zimmerman MD, Prideaux B, Dartois V, et al. Statin adjunctive therapy shortens the duration of TB treatment in mice. J Antimicrob Chemother. (2016) 71:1570-7. doi: 10.1093/jac/dkw014

30. Bruiners N, Dutta NK, Guerrini V, Salamon H, Yamaguchi KD, Karakousis PC, et al. The anti-tubercular activity of simvastatin is mediated by cholesterol-dependent regulation of autophagy via the AMPK-mTORC1TFEB axis. bioRxiv [Preprint]. (2020). doi: 10.2139/ssrn.3552811 
31. Lai CC, Lee MT, Lee SH, Hsu WT, Chang SS, Chen SC, et al. Statin treatment is associated with a decreased risk of active tuberculosis: an analysis of a nationally representative cohort. Thorax. (2016) 71:646-51. doi: 10.1136/thoraxjnl-2015-207052

32. Su VY, Su WJ, Yen YF, Pan SW, Chuang PH, Feng JY, et al. Statin use is associated with a lower risk of TB. Chest. (2017) 152:598-606. doi: 10.1016/j.chest.2017.04.170

33. Baigent C, Keech A, Kearney PM, Blackwell L, Buck G, Pollicino C, et al. Efficacy and safety of cholesterol-lowering treatment: prospective metaanalysis of data from 90,056 participants in 14 randomised trials of statins. Lancet. (2005) 366:1267-78. doi: 10.1016/S0140-6736(05)67394-1

34. Karp I, Chen SF, Pilote L. Sex differences in the effectiveness of statins after myocardial infarction. CMAJ. (2007) 176:333-8. doi: 10.1503/cmaj.060627

35. Zissimopoulos JM, Barthold D, Brinton RD, Joyce G. Sex and race differences in the association between statin use and the incidence of Alzheimer Disease. JAMA Neurol. (2017) 74:225-32. doi: 10.1001/jamaneurol.2016.3783

36. Ohtawa M, Uchiyama N. Sex difference in metabolism of simvastatin by rat hepatic microsomes. Eur J Drug Metab Pharmacokinet. (1992) 17:175-81. doi: 10.1007/BF03190142

37. Vree TB, Dammers E, Ulc I, Horkovics-Kovats S, Ryska M, Merkx I. Differences between lovastatin and simvastatin hydrolysis in healthy male and female volunteers:gut hydrolysis of lovastatin is twice that of simvastatin. ScientificWorldJournal. (2003) 3:1332-43. doi: 10.1100/tsw.2003.121

38. Nakajima K. Sex-related differences in response of plasma lipids to simvastatin: the saitama postmenopausal lipid intervention study. SPOLIS Group Clin Ther. (1999) 21:2047-57. doi: 10.1016/S0149-2918(00) 87236-7

39. Vilaplana C, Marzo E, Tapia G, Diaz J, Garcia V, Cardona PJ. Ibuprofen therapy resulted in significantly decreased tissue bacillary loads and increased survival in a new murine experimental model of active tuberculosis. J Infect Dis. (2013) 208:199-202. doi: 10.1093/infdis/jit152

Conflict of Interest: The authors declare that the research was conducted in the absence of any commercial or financial relationships that could be construed as a potential conflict of interest.

Copyright (c) 2020 Dutta and Schneider. This is an open-access article distributed under the terms of the Creative Commons Attribution License (CC BY). The use, distribution or reproduction in other forums is permitted, provided the original author(s) and the copyright owner(s) are credited and that the original publication in this journal is cited, in accordance with accepted academic practice. No use, distribution or reproduction is permitted which does not comply with these terms. 